\title{
Correlation between histocompatibility antigens and recurrent aphthous stomatitis in the brazilian population
} Niels Salles Willo Wilbelmsen', Raimar Weber ${ }^{2}$,
Francisco Monteiro ${ }^{3}$, Jorge Kalil', Ivan Dieb
Miziara $^{5}$
Keywords: hla antigens, aphthous stomatitis, genetics, stomatology.

\section{Summary}

\begin{abstract}
$\mathrm{R}$ Recurrent aphthous stomatitis is a common oral mucosa disorder that affects $20 \%$ of the world's population, characterized by recurring painful ulcers in the mouth. The diagnosis is primarily based on the patient's clinical history. Inheritance may pose as a risk factor for the disease; however, the studies available are inconclusive as to the results attained, and they vary according to the population studied. Aim: to typify class I and class II HLA molecules and to assess how frequent these molecules are present in the Brazilian population with Recurrent Aphthous Stomatitis, compared to healthy controls. Materials and Methods: In this prospective, cross-sectional and investigative study, thirty one patients with diagnostic hypothesis of recurrent aphthous stomatitis were seen from February of 2004 to May of 2006. We obtained the DNA from those patients who matched the inclusion criteria and typified their HLA by PCR. Results: In those patients with Recurrent Minor Aphthous Stomatitis we found statistically significant occurrences of HLA-A33 and HLA-B35. Conclusion: HLA-A33 and HLA-B35 may be associated with recurrent minor aphthous stomatitis in the Brazilian's population.
\end{abstract}

\footnotetext{
${ }^{1} \mathrm{PhD}$ in Otorhinolaryngology.

${ }^{2}$ Graduate Student $(\mathrm{PhD})$ in Otorhinolaryngology.

${ }^{3} \mathrm{PhD}$ in Nephrology, Professor.

${ }^{4}$ Full Professor of Immunology and Clinical Allergy of the University of São Paulo Medical School.

${ }^{5}$ Associate Professor of Otorhinolaryngology.

University of São Paulo Medical School.

Send correspondence to: Niels Salles Willo Wilhelmsen - Rua Doutor Messuti 136 apto. 92 Vila Bastos Santo André SP 09041-160. State of São Paulo Research Support Foundation - FAPESP (Process \# 05/51085-0).

Paper submitted to the BJORL-SGP (Publishing Management System - Brazilian Journal of Otorhinolaryngology) on January 16, 2008; and accepted on August 11, 2008. cod. 5680
} 


\section{INTRODUCTION}

Recurrent Aphthoid Stomatitis (RAS) is a common disease that causes the appearance of recurrent aphtha in the oral mucosa. Its incidence is of about $20 \%$ in the world population. It is more frequent in females. Despite the high incidence, and many studies dedicated to unveiling its causes, it still is very controversial regarding etiology ${ }^{1}$.

RAS is currently defined as a disease characterized by aphtha lesions in the oral mucosa, in a recurrent fashion (every fortnight or monthly), for a minimum period of one year, and its onset is usually in childhood or adolescence ${ }^{2}$, without evidences of associated systemic diseases. Nonetheless, some authors state that the incidence of RAS is greater in the second decade of life $e^{3,4}$.

The disease usually has three clinical forms, based on the aspect and size of the ulcerations: minor, major and herpetiform. Recurrent Aphthoid Stomatitis Minor (RASi) affects $80 \%$ of the patients with RAS, and it is characterized by a shallow painful, oval or round-shaped ulcer, smaller than $5 \mathrm{~mm}$ in diameter. It affects the non-keratinized oral mucosa, especially vestibule, labial and oral mucosa, soft palate, tongue and tongue floor. The lesions heal within 7 to 10 days. Recurrent Aphthoid Stomatitis Major (RASj) is the most severe form. Aphtha diameter can get bigger than $1 \mathrm{~cm}$, remaining in the oral cavity for a period longer than two weeks, often times leaving a scar. The third form of the disease, and the less common one is Herpetiform Recurrent Aphthoid Stomatitis (HRAS), characterized by multiple and painful 1 to $3 \mathrm{~mm}$ ulcers, which may coalesce, forming larger and irregular lesions 5 .

Family history seems to be relevant in the genesis of RAS, and reports of cases in the same family are found in $24 \%$ to $46 \%$ of the times ${ }^{6,7}$. Moreover, patients with family history of RAS can develop ulcers earlier on and have more severe manifestations than those without family history ${ }^{8}$. Monozygotic twins have a greater likelihood of suffering the disease when compared to dizygotic twins?

Numerous associations and non-associations of HLA and RAS antigen have been reported in the medical literature. The association between the disease and HLAB12 was described by Lehner et al. ${ }^{10}$ and Malmström et al.11, however it was not confirmed by other authors ${ }^{12,13}$. In groups of patients of different ethnical origin, a significant association between HLA-DR2 and RAS was noticed ${ }^{10,14}$.

RAS pathophysiology seems to be associated with a disorder in immunomodulation ${ }^{15}$. Lymphocytes seem to be the predominant cells in aphthoid lesions, and there was a variation in the CD $4+/ \mathrm{CD} 8+$ ratio during its different stages - prodrome or pre-ulceration, ulceration and healing ${ }^{16,17,18}$.

The main role played by the major histocompatibility complex in humans, called HLA system is to present processed peptides for recognition by the $\mathrm{T}$-cell receptor in the different immunological functions. Since such recognition is made at the same time involving the peptide and the HLA molecule, small variations in one of the two components cause immunologic recognition and the triggering of cell activation and a specific response ${ }^{19}$. It is so understood because the HLA molecules are directly implicated in the genetic control of the immune response.

We do not know of any Brazilian study that associates HLA type with RAS. Having said that, the goal of the present investigation was to typify classes I and II of HLA molecules from Brazilian patients with RAS and compare with the control group made up of 964 healthy Brazilians.

\section{MATERIALS AND METHODS}

\section{Material}

This is a prospective, cross-sectional, investigative study, carried out from February of 2004 through May of 2006. It was approved by the Ethics in Research Committee of the University of São Paulo Medical School, under protocol \# 876/04.

\section{Study Group}

The study group was initially made up of 58 patients with diagnostic suspicion of RAS, seen at the Stomatology Division of the ETN Clinic of the University of São Paulo Medical School Hospital.

\section{Control Group}

The control group was made up by a selection of 4172 healthy Brazilian volunteers, members of the bone marrow donors data bank of the Transplant Center of the University of São Paulo Medical School Hospital.

From these, we selected only one member of each family, in order to avoid the presence of haplotypes, at the end making up a total of 962 Brazilian individuals.

\section{Method}

The patients were submitted to an interview, general physical exam, complete otorhinolaryngological exam, and a previous workup protocol, besides biopsies of the lesion and adjacent mucosa.

The prior workup protocol involved fast glucose, protein electrophoresis, CBC, coagulogram (TP + TT + TTPA), anti-HIV 1 and 2, dosage of total complement and fractions, rheumatoid factor, antinucleus factors, serum dosage of IgA, IgG, IgM, VHS, C-Reactive protein and serology for syphilis.

In order to rule out the etiology of ulcers associated with other diseases, such as pemphigoid lesions, Behcet's disease, Crohn's disease or vasculitis of other nature, all the patients were submitted to lesion biopsy for later histopathology test and direct immunofluorescence test. 
Biopsy was carried out under 2\% xylocaine injection without vasoconstrictor, by means of a puncher the size of the lesion (4-5mm). Two samples were taken; one with the lesion and one with the healthy adjacent area, the former was placed in a flask with 10\% formaldehyde and sent to histopathological exam. The second sample was placed on gauze soaked in saline solution and immediately referred to the Skin Immunology Division of the Department of Dermatology for direct immunofluorescence.

\section{Inclusion and Exclusion Criteria}

In the study we included all those patients who reported at least on episode of aphtha (s) per month for a minimum period of one year, as well as those who signed the informed consent form agreeing with the procedures employed.

We excluded those patients who had alterations in the serum exam or those whom in the histopathology and direct immunofluorescence study had alterations that characterize other diseases. These patients were referred to specific treatment of the alterations found.

In order to obtain DNA, we collected $10 \mathrm{ml}$ of total peripheral blood in an EDTA $25 \mathrm{mM}$ flask. DNA extractions were carried out by the Hexadecyltrimethylammonium Bromide/dodecyltrimethylammonium Bromide (DTAB/ CTAB) test and HLA class I and II types by chain polymerization - specific oligonucleotide sequence (PCR-SSO). The analysis was carried out in the Heart Institute of the University of São Paulo Medical School Hospital (Incor FMUSP).

Blood collection, DNA extraction and HLA typing of both classes from the control group had already been carried out in this same lab.

\section{Statistical Analysis}

In order to check whether or not there was any association between HLA antigens and their subgroups and RAS disease, we used the Chi-Squared test, and a given association was considered statistically significant when the test's description level (p) were lower than 0.05 (or 5\%). In order to estimate the power of the associations found, we calculated the relative risks $(\mathrm{RR})$ and their respective confidence intervals of 95\% (CI 95\%).

The data was analyzed using the Statistical Package for Social Sciences (SPSS ${ }^{\circledR}$ for Windows 10.0; SPSS Inc, Chicago, Illinois, USA) software.

\section{RESULTS}

Of the 58 initial patients, 27 were taken off the study for numerous reasons. Of the remaining 31 patients, 17 (54.84\%) were women, 14 (45.16\%) were men. Age varied between 7 and 70 years, reaching an average of 36.14 years and standard deviation of 15.33 (Table 1).

As to the types of aphthas the study patients had, there was a predominance of the minor type (67.74\%). Please notice the absence of patients with herpetiform aphthas (Table 1).

Regarding the frequency type of Class I an II HLA antigens in the study group, Table 2 shows the frequency of each patient.

Table 3 shows the prevalences of HLA Class I antigen in which there was a statistically significant difference between the study group (without considering the type of aphtha) and the control group. The relative risk (RR) of patients with RAS who had HLA frequencies of A33, $\mathrm{B} 35$ and $\mathrm{B} 81$ was of 3.48 (CI95\% 1.38 - 8.8; $\mathrm{p}=0.016)$; 3.48 (CI95\% $1.69-7.16 ; \mathrm{p}<0.001)$ and 8.22 (CI95\% 1.67 - 40.41; $\mathrm{p}=0.036$ ), respectively, times the control group.

Class II HLA frequencies of the study group which had greater index were Dr7, present in 10 patients (16.13\%), Dr11 and Dr 17, the latter two were present in eight patients (12.90\%). When compared to the control group, there was no statistically significant difference.

When RAS patients were broken down according to the type of aphtha, the prevalence of HLA A33 and

Table 1. Demographic data from the 31 patients with RAS according to the type of RAS presented.

\begin{tabular}{|c|c|c|c|c|c|}
\hline & Aphtha type & & & & \\
\hline & Minor & Major & Herpetiform & $p$ & Total \\
\hline & $(n=21)$ & $(n=10)$ & $(n=0)$ & & $(n=31)$ \\
\hline & $(67,74 \%)$ & $(32,26 \%)$ & $(0,00 \%)$ & & $(100,00 \%)$ \\
\hline \multicolumn{6}{|l|}{ Gender } \\
\hline Male & $10(47,6 \%)$ & $4(40,0 \%)$ & $0(0,0 \%)$ & 1,0 & $14(45,2 \%)$ \\
\hline Female & $11(52,4 \%)$ & $6(60,0 \%)$ & $0(0,0 \%)$ & & $17(54,8 \%)$ \\
\hline Age (years) & $35,6 \pm 14,5$ & $37,2 \pm 17,5$ & - & 0,79 & $36,1 \pm 15,2$ \\
\hline$<20$ & $2(9,5 \%)$ & $2(20,0 \%)$ & & 0,35 & $4(12,9 \%)$ \\
\hline 20 a 39 & $12(57,1 \%)$ & $3(30,0 \%)$ & & & $15(48,4 \%)$ \\
\hline$\geq 40$ & $7(33,3 \%)$ & $5(50,0 \%)$ & & & $12(38,7 \%)$ \\
\hline
\end{tabular}


Table 2. Distribution as to HLA class I and II frequency of 31 patients with RAS.

\begin{tabular}{|c|c|c|c|}
\hline Patient & HLA-A & HLA-B & HLA-Dr \\
\hline 1 & A1 A11 & B35 B63 & DR13 DR16 \\
\hline 2 & A23 A26 & B50 B51 & DR4 DR13 \\
\hline 3 & A24 A24 & B35 B65 & DR1 DR16 \\
\hline 4 & A25 A34 & B7 B42 & DR7 DR10 \\
\hline 5 & A33 A68 & B65 B71 & DR1 DR 17 \\
\hline 6 & $\mathrm{~A} 2 \mathrm{~A} 2$ & B7 B61 & DR10 DR11 \\
\hline 7 & A2 A32 & B18 B35 & DR4 DR16 \\
\hline 8 & A2 A66 & B35 B58 & DR13 DR17 \\
\hline 9 & A74 A74 & B35 B81 & DR9 DR14 \\
\hline 10 & A3 A29 & B35 B44 & DR8 DR7 \\
\hline 11 & A1 A24 & B13 B56 & DR1 DR15 \\
\hline 12 & A23 A80 & B44 B72 & DR4 DR7 \\
\hline 13 & A24 A33 & B35 B49 & DR7 DR15 \\
\hline 14 & A24 A29 & B51 B64 & DR7 DR11 \\
\hline 15 & A2 A23 & B50 B51 & DR7 DR11 \\
\hline 16 & A2 A30 & B18 B39 & DR14 DR17 \\
\hline 17 & A32 A66 & B35 B58 & DR4 DR11 \\
\hline 18 & $\mathrm{~A} 11 \mathrm{~A} 11$ & B7 B35 & DR15 DR103 \\
\hline 19 & A2 A24 & B18 B35 & DR8 DR17 \\
\hline 20 & $\mathrm{~A} 1 \mathrm{~A} 2$ & B37 B50 & DR7 DR103 \\
\hline 21 & $\mathrm{~A} 1 \mathrm{~A} 3$ & B35 B57 & DR11 DR17 \\
\hline 22 & A1 A23 & B18 B81 & DR7 DR11 \\
\hline 23 & A24 A31 & B35 B51 & DR11 DR13 \\
\hline 24 & A2 A29 & B44 B64 & DR7 DR17 \\
\hline 25 & $\mathrm{~A} 2 \mathrm{~A} 3$ & B51 B62 & DR13 DR16 \\
\hline 26 & А31 А33 & B39 B65 & DR1 DR8 \\
\hline 27 & A24 A30 & B8 B35 & DR11 DR17 \\
\hline 28 & АЗ АЗ3 & B35 B49 & DR7 DR7 \\
\hline 29 & A11 A33 & B13 B35 & DR15 DR15 \\
\hline 30 & A2 A33 & B41 B65 & DR1 DR17 \\
\hline 31 & A2 A24 & B18 B62 & DR8 DR15 \\
\hline
\end{tabular}

Table 3. Class I HLA antigen frequency in patients with Recurrent Aphthoid Stomatitis (RAS) compared to the control group.

\begin{tabular}{|c|c|c|c|c|}
\hline & Group & & & \\
\hline & $\begin{array}{c}\text { RAS } \\
(n=31)\end{array}$ & $\begin{array}{c}\text { Control } \\
(\mathrm{n}=961)\end{array}$ & $p$ & $\begin{array}{c}\text { RR } \\
\text { (Cl 95\%) }\end{array}$ \\
\hline A33 & $6(19,4 \%)$ & $62(6,5 \%)$ & 0,016 & $3,48(1,38-8,80)$ \\
\hline B35 & $15(48,4 \%)$ & $204(21,2 \%)$ & $<0,001$ & $3,48(1,69-7,16)$ \\
\hline B81 & $2(6,5 \%)$ & $8(0,8 \%)$ & 0,036 & $8,22(1,67-40,41)$ \\
\hline
\end{tabular}

$\mathrm{RR}=$ Relative Risk; $\mathrm{Cl}=$ Confidence Interval
B35 frequencies in the group with aphtha minor remained higher than those from the control group, in a statistically significant way, with RR of 3.27 (1.1 - 9.4; p = 0.047 ) and 4.73 (CI95\% $2.0-11.1 ; \mathrm{p}<0.001)$, in relation to the control group, respectively, according to Table 4 . Because of the reduction in the number of patients after the subdivision was made, the differences in HLA B81 frequency prevalences between both groups of aphtha and control patients, and HLA A33 and B35 between the aphtha major and control groups have a greater likelihood (5\%) of being by chance and, therefore, not statistically significant $(\mathrm{p}>0.05)$.

Table 4. Class I HLA antigen frequency in patients with major and minor Recurrent Aphthoid Stomatitis compared to the Control Group.

\begin{tabular}{|c|c|c|c|c|}
\hline \multicolumn{5}{|c|}{ Group } \\
\hline & $\begin{array}{l}\text { Aphtha } \\
\text { Minor } \\
(n=21)\end{array}$ & $\begin{array}{l}\text { Controls } \\
(\mathrm{n}=961)\end{array}$ & $p$ & $\mathrm{RR}(\mathrm{Cl} 95 \%)$ \\
\hline A33 & $4(19,0 \%)$ & $62(6,5 \%)$ & 0,047 & $3,27(1,1-9,4)$ \\
\hline B35 & $12(57,1 \%)$ & 204 (21,2 \%) & $<0,001$ & $4,73(2,0-11,1)$ \\
\hline B81 & $1(4,8 \%)$ & $8(0,8 \%)$ & 0,177 & $5,41(0,8-36,1)$ \\
\hline \multicolumn{5}{|c|}{ Group } \\
\hline & $\begin{array}{c}\text { Aphtha } \\
\text { Major } \\
(n=10)\end{array}$ & $\begin{array}{l}\text { Controls } \\
(\mathrm{n}=961)\end{array}$ & $\mathrm{p}$ & RR (Cl 95\% \\
\hline A33 & $2(20,0 \%)$ & $62(6,5 \%)$ & 0,137 & $3,54(0,8-16,3)$ \\
\hline B35 & $3(30,0 \%)$ & 204 (21,2 \%) & 0,452 & $1,58(0,4-6,1)$ \\
\hline B81 & $1(10,0 \%)$ & $8(0,8 \%)$ & 0,089 & $11,88(1,7-84,2)$ \\
\hline
\end{tabular}

$\mathrm{RR}=$ Relative Risk; $\mathrm{Cl}=$ Confidence Interval

\section{DISCUSSION}

Recurrent aphthoid stomatitis is a common disease in the oral cavity and its etiology is still undefined ${ }^{20,3,7}$. It may be associated with local and systemic factors, its origin is immunological, microbiological and genetic ${ }^{21,22}$.

According to Jurge et al. ${ }^{4}$, the incidence of RAS is more common in the second decade of life, between 20 and 30 years of age. In our study, the incidence was also higher in this same age range, with a total of eight patients (25.80\%). Values very near this one, seven (22.5\%), were obtained in the two following age ranges (30 to 40 years and 40 to 50 years).

Females had the higher RAS prevalence, 54.84\%, when compared to males, $45.16 \%$. These data are in agreement with the findings by Axéll and Henricsson23, but are also in conflict with those found by Miller et al. ${ }^{\text {, }}$ in which males had a higher prevalence.

In our study, the HLA frequencies that had the highest rates of patients with RAS were: HLA-A33, HLA- 
B35 and HLA-B81. When seen under the light of disease subtype, we found significant values for aphtha minor, in the frequencies of HLA-A33 and HLA-B35. For RAS Major, no frequency presented statistically significant value.

In an English population, Challacombe et al. ${ }^{18}$ found the HLA-B12 frequence associated with patients with RAS. When analyzed in relation to the subtypes of aphthoid Stomatitis the same B12 frequency was statistically significant only for herpetiform aphthas. For types Major and Minor, no prevalent frequency was found by the authors. In our study, because of its low incidence, it was not possible to find patients with the herpetiform type.

The same HLA-B12 frequency was found by Malmström et al. ${ }^{11}$ when they studied 14 Finish patients with RAS. Differently from our studies, these authors did not use a control group, and their RAS patient sample was small.

Gallina et al. ${ }^{12}$, with a sample of 26 Sicilian patients with RAS, found a statistically increased value for HLA-DR7 frequency. As we make a critical analysis of the experimental group selected, we notice that patients with only two episodes per year were considered RAS patients, and such characteristic did not match the RAS clinical picture established by Stanley ${ }^{5}$ and Scully ${ }^{2}$, who characterized as RAS those monthly episodes of aphtha for at least one year, the same we did in our study.

Albanidou-Farmaki et al. ${ }^{14}$ did a study with 106 patients with RAS. The HLA-DR5 frequency, differently from our findings, was predominant in these patients. On the other hand, the HLA-DR4 frequency was reduced.

Sun et al. ${ }^{24}$ stated that the HLA-DRw9 frequency can be considered a genetic marker for RAS in the Chinese population. Their study was made up of 80 Chinese patients with RAS. In this study, they typed the HLA-DR, differently from our study, in which we also typed the HLA-A and B.

In an Arab population, Jaber et al. ${ }^{25}$ studied 22 patients with RAS, and observed a statistically significant increase in HLA-B52 and B44 molecules in patients with this disease when compared to the control group. The HLA-B51 frequency has been found in some papers associated with RAS in countries like Israel and Korea ${ }^{26,27}$.

It is important to stress that with the progress in HLA typing methodology, the serum method is no longer used, in which it was necessary to have specific ligants (antibodies) in order to show the presence of the corresponding HLA frequence, as used by some authors ${ }^{11,12,14,18,28,29}$. In our study, we used the cellular method, in which one uses the mixed lymphocitic reactions, making the HLA typing more reliable, allowing the discovery of new frequencies ${ }^{30}$.

On the other hand, according to Braun Prado et al. ${ }^{31}$ and Monte et al. ${ }^{32}$, the HLA-B35 frequency is extremely common in the general Brazilian population, and such fact was also seen in our control group. Nonetheless, it is important to stress that although the HLA-B35 frequency was also found high in our experimental group; such fact did not happen by chance, there was a statistically significant difference in the incidence of this gene between the two groups.

We must also remember that, as mentioned by Louzada-Junior et al. ${ }^{33}$ the reduced frequency of HLA typing could be a protection factor for RAS. In our study we did not notice any of such events.

Finally, the fact that many papers found HLA frequencies for RAS different from the ones found in our study, probably happened because the populations studied were from different nationalities (Danish, Scottish, Israelis, Chinese, Koreans) and ethnic origins different from ours, a highly mixed races country.

Our findings show that the HLA frequencies must be done specifically for each population, so that we can map the possible genetic factors involved in the genesis of this disorder.

We have to stress in our study that the lack of patients with herpetiform recurrent aphthoid stomatitis and the low number of patients with stomatitis type major, prevented a more accurate assessment of the HLA frequencies associated with these disease forms.

At any rate, the relatively reduced series of patients with RAS in our study does not invalidate our results, having seen that the HLA frequencies found in our patients were very different from those obtained from the control group of patients without RAS.

\section{CONCLUSION}

The results from our study suggest that minor RAS is associated with the HLA-A33 and HLA-B35 frequencies in the Brazilian population.

\section{ACKNOWLEDGEMENT}

This paper counted on the financial support from the FAPESP - Fundação de Amparo a Pesquisa do Estado de São Paulo - FAPESP (Process \# 05/51085-0).

\section{REFERENCES}

1. Natah SS, Konttinen YT., Enattah NS, Ashammakhi N, Sharkey KA, Hayrinen-Immonen R. Recurrent aphthous ulcera today: a review of the growing knowledge. Int J Oral Maxillofac Surg. 2004;33(3): 221-34.

2. Scully C. Aphthous Ulceration. N Eng J Med 2006; 355(2):165-72.

3. Porter SR, Hegarty A, Kaliakatsou F, Hodgson T, Scully C. Recurrent aphthous stomatitis. Clinics in Dermatol. 2000;18:569-78.

4. Jurge S, Kuffer R, Scully C, Porter SR. Recurrent aphthous stomatitis. Oral Diseases. 2006;12:1-21.

5. Stanley HR. Aphthous lesions. Oral Surg Oral Med Oral Pathol. 1972;33(3):407-16.

6. Sircus W, Church R, Kelleher J. Recurrent aphthous ulceration of the mouth; a study of the natural history, aetiology and treatment. Q J Med. 1957;26(102):235-49. 
7. Casiglia JM. Recurrent aphthous stomatitis: Etiology, diagnosis, and treatment. General Dentistry. 2002;50(2):157-166.

8. Ship II. Inhertance of Aphthous ulcers of the mouth. Oral Surg Oral Med Oral Pathol. 1965;44(5):837-44

9. Miller MF, Ship II, Ram C. A retrospective study of factors associated with recurrent aphthous ulcers in a professional population. Oral Surg. 1977;43:532-7.

10. Lehner T, Welsh KL, Batchelor JR. The relationship of HLA-B and DR phenotypes to Behçet's syndrome, recurrent oral ulceration and the class of immune complexes. Immunology. 1982;47,581-7.

11. Malmström M, Salo OP, Fyhrquist F. Immunogenetic markers and immune response in patients with recurrent oral ulceration. Int J Oral Surg. 1983;12:23-30

12. Gallina G, Cumbo V, Messina P, Caruso C. HLA-A, B, C, DR, MT, and $\mathrm{MB}$ antigens in recurrent aphthous stomatitis. Oral Surg Oral Med Oral Pathol. 1985;59(4):364-70.

13. Ozbakir F, Yazici H, Mat C, Tuzun Y, Yurdakul S, Yilmazer S. HLA antigens in recurrent oral ulceration: evidence against a common disease spectrum with Behcet's syndrome. Clin Exp Rheumatol. $1987 ; 5: 263-5$.

14. Albanidou-Farmaki A, Kayavis IG, Polymenidis Z, Papanayotou P. HLA-A, B, C na DR Antigens in Recurrent Oral Ulcers. Ann Dent. 1988; $47(1): 5-8$.

15. Sistig S, Cekic-Arambasin A, Rabatic S, Vucicevic-Boras V, Kleinheinz J, Piffko J. Natural immunity in recurrent aphthous ulceration. J Oral Pathol Med. 2001;30:275-80.

16. Greenspan JS, Gadol N, Olson JA, Hoover CI, Jacobsen PL, Shillitoe EJ et al. Lymphocyte function in recurrent aphthous ulceration. J Oral Pathol. 1985;14:592-6.

17. Pedersen A, Klausen B, Hougen HP, Ryder LP. Peripheral lymphocyte subpopulations in recurrent aphthous ulceration. Acta Odontol Scand. 1991;49:203-6.

18. Challacombe SJ, Barkhan P, Lehner T. Haematological features and differentiation of recurrent oral ulceration. Br J Oral Surg. 1977;15: 37-48.

19. Janeway CA, Travers P, Walport M, Shlomchik M. Immunobiology: the immune system in health and disease. $5 \mathrm{a}$ ed. Garland Publishing 2001.
20. Porter SR, Scully C, Pedersen A. Recurrent aphthous stomatitis. Crit Rev Oral Biol Med. 1998;9(3):306-21.

21. Scully C. Oral infections in the immunocompromised patient. $\mathrm{Br}$ Dent. J 1992;172(11):401-7.

22. Miziara, ID. Estomatite Aftoide Recidivante. Rev Bras de Otorrinolaringol. 1995; 61(5):418.

23. Axéll T, Henricsson V. The occurrence of recurrent aphthous ulcers in an adult Swedish population. Acta Odontol Scand. 1985;43:121-5.

24. Sun A, Hsieh RO, Chu CT, Wu YC. Strong association of HLA-DRw9 in Chinese patients with recurrent oral ulcers. J Am Acad Dermatol. 1991;24(2 Pt 1):195-8.

25. Jaber L, Weinberger A, Klein T, Yaniv I, Mukamel M. Close association of HLA-B52 and HLA-B44 antigens in Israeli Arab adolescents with recurrent aphthous stomatitis. Arch Otolaryngol Head Neck Surg. 2001;127:184-7.

26. Shohat-Zabarski R, Kalderon S, Klein T, Weinberger A. Close association of HLA-B51 in persons with recurrent aphthous stomatitis Oral Surg Oral Med Oral Pathol. 1992;74(4):455-8.

27. Chang HK, Kim JU, Cheon KS, Chung HR, Lee KW, Lee IH. HLA-B51 and its allelic types in association with Behçet's disease and recurrent stomatitis in Korea. Clin Exp Rheumatol. 2001;19 (24):S31-S35.

28. Platz P, Ryder LP, Donatsky O. No deviations of HLA-A and -B Antigens in patients with Recurrent Aphtous Stomatitis. Tissue Antigens. 1976;8:279-80.

29. Dolby AE, Walker DM, Slade M, Allan C. HL-A histocompatibility antigens in recurrent aphthous ulceration. J Dent Res. 1977;56(2):105-7.

30. Bodmer JG, Marsh SGE, Albert ED, Bodmer WF, Dupont B, Erlich HA, Mach B et al.. Nomenclature for factors of the HLA system, 1998. Tissue Antigens. 1999;53:407-68.

31. Braun-Prado K, Mion ALV, Culpi L, Petzi-Erler ML. HLA class I polymorphism, as characterised by PCR-SSOP, in Brasilian exogamic population. Tissue Antigens. 2000;56:417-27.

32. Monte SJH, Neto JMM, Rampim GF, Shulzhenko N, Morgun A, Delima MG. Polimorfismo do sistema HLA em uma amostra de mestiços da população de Teresina, Piauí. Rev Assoc Med Bras. 2004;50(4):422-6.

33. Louzada-Junior P, Smith AG, Hansen JA, Donadi EA. HLA-DRB1 and -DQB1 alleles in the Brazilian population of the northeastern region of the state of São Paulo. Tissue Antigen. 2001;57:158-62. 